\title{
Rúbricas de evaluación y autoevaluación: una estrategia útil para identificar el nivel de comprensión de conceptos químicos en estudiantes de grado $10 .^{\circ}$
}

\author{
Evaluation and Self-Assessment Rubrics: A Useful \\ Strategy to Identify the Level of Understanding of \\ Chemical Concepts in Tenth-Grade Students
}

\author{
Laura Cárdenas ${ }^{1}$
}

\section{Resumen}

En este artículo se plantea el desarrollo de un proyecto realizado con el fin de establecer la importancia de la autoevaluación dentro del proceso de evaluación realizado en las clases de química del grado $10 .^{\circ}$ del colegio Champagnat de Bogotá. Es importante centrar la atención en la evaluación pues es finalmente mediante este proceso que el docente y el estudiante se informan del nivel de comprensión que se ha adquirido, para así tomar medidas frente a su proceso y plantear nuevas estrategias que permitan alcanzar los desempeños propuestos. Cuando el estudiante es consciente de su proceso de comprensión, es capaz de plantearse nuevos retos y llegar a evaluar a sus compañeros bajo criterios en los que las rúbricas de evaluación juegan un papel importante. Durante el transcurso de la Práctica I se evidenciaron algunas dificultades de los estudiantes al momento de desarrollar la evaluación diagnóstica, lo que no era coherente con los resultados del trabajo en clase y los procesos de autoevaluación que daban a entender que se comprendían los conceptos estudiados. Además, durante el proceso de autoevaluación, la mayoría de veces los estudiantes no eran conscientes de su propio proceso de aprendizaje, sino que el resultado de esta se asumía como una oportunidad para mejorar la nota. Lo anterior generó las siguientes preguntas: ¿sé estará promoviendo una evaluación diagnóstica continua en el grado $10 .^{\circ}$ del colegio Champagnat, donde el estudiante identifica su comprensión de los conceptos químicos a partir de un proceso de autoevaluación? ¿Mediante la implementación de las rúbricas de evaluación mejorarán los procesos de autoevaluación en los estudiantes al permitir que participen tomando conciencia sobre su proceso de

1 Profesora en formación, Universidad Pedagógica Nacional. Correo electrónico: laucardenas20@gmail.com 
comprensión? El desarrollo de la investigación planteó la rúbrica de evaluación, la cual permitió realizar un proceso riguroso del nivel de comprensión de los estudiantes, desde los procesos tanto de heteroevaluación y coevaluación como de autoevaluación.

Palabras clave

enseñanza para la comprensión, evaluación, autoevaluación, rúbrica.

\begin{abstract}
This article considers the development of a project carried out in order to establish the importance of self-assessment within the evaluation process carried out in tenth-grade chemistry classes at the Champagnat School in Bogotá. It is important to focus attention on the evaluation because it is ultimately through this process that the teacher and the student are informed of the level of understanding acquired, in order to take action about their process and propose new strategies to achieve the proposed performances. When students are aware of their comprehension process, they are able to consider new challenges and to get to evaluate his classmates under criteria in which the evaluation rubrics play an important role. During the Practice I course, students revealed some difficulties when developing the diagnostic assessment, which was not consistent with the results of the classwork and the self-assessment processes that suggested that the concepts studied were understood. In addition, during the self-assessment process, most of the time students were not aware of their own learning process, but the result of this was assumed as an opportunity to improve the grade. This generated the following questions: is a continuous diagnostic evaluation in the $10^{\text {th }}$ grade class of the Champagnat School, where the student identifies his or her understanding of the chemical concepts from a self-assessment process being promoted? Through the implementation of the evaluation rubrics, will students' self-assessment processes improve by allowing them to participate, becoming aware of their comprehension process? The development of the research raised the rubric of evaluation, which made it possible to carry out a rigorous process of the level of understanding of the students, from the processes of hetero-assessment and co-assessment as well as of self-assessment.
\end{abstract}

Keywords

teaching for understanding, evaluation, self-assessment, rubric. 


\section{Introducción}

Evaluar en comprensión tiene como objetivo mirar la capacidad del estudiante para comprender y analizar el concepto en diferentes ámbitos; este proceso no solo va ligado a la evaluación que el docente realiza, sino, por el contrario, toma como apoyo la autoevaluación que realiza el estudiante, pues al evaluar su proceso de comprensión identifica oportunidades de mejoramiento, y fortalezas propias y de sus compañeros. Stone (1999,p. 14), plantea que "al involucrar a los estudiantes en la evaluación de su propio trabajo y el de sus compañeros de clase, los invita a hacerse más responsable de su propio aprendizaje". Para apoyar este proceso de autoevaluación se implementa la rúbrica, la cual es una matriz de valoración en la que se plasma un conjunto de criterios específicos que permiten valorar el aprendizaje de los conocimientos y las competencias adquiridas; el uso de rúbricas permite que el estudiante pueda participar tomando conciencia sobre la comprensión de las temáticas para plantear nuevas metas y retos académicos.

El proyecto tuvo como fin diseñar e implementar rúbricas que permitieran identificar los procesos de comprensión alcanzados por los estudiantes de grado $10 .^{\circ}$ del colegio Champagnat durante el segundo semestre del 2016. Con ayuda de las rúbricas no solo se establecieron criterios de evaluación, sino que se pudo evidenciar un avance en los conceptos y temas trabajados. El estudiante fue consciente de los procesos que se realizaron y mediante su autoevaluación corroboró el nivel de comprensión alcanzado en cada uno de los temas. Adicionalmente, la rúbrica de evaluación permitió establecer una relación entre la prueba diagnóstica y las actividades de aprendizaje que se desarrollaron.

Se llevaron a cabo procesos de observación, caracterización y diseño de un instrumento que permitió orientar las ideas que se generaron durante la Práctica I y que despertaron un interés por los procesos de autoevaluación que realizaban los estudiantes de grado $10 .^{\circ}$ del colegio Champagnat. Por otro lado, los resultados obtenidos en los procesos de autoevaluación y la evaluación diagnóstica realizados eran contradictorios: mientras en la autoevaluación el estudiante reflejó tener dominio de la mayoría de los temas abordados en la clase, en la evaluación diagnóstica el estudiante mostró un bajo desempeño; esto ocurrió porque los estudiantes a la hora de realizar los procesos de autoevaluación no eran conscientes de su proceso de comprensión, sino de la repercusión del valor cuantitativo en la nota final. Por este motivo, en el segundo semestre del 2016 se plantearon las rúbricas de evaluación que, mediante criterios, permitieron dar a conocer el nivel de comprensión alcanzado por los estudiantes; los criterios que se plasmaron se encuentran propuestos en el Sistema Institucional de Evaluación de Estudiante - SIEE del Colegio Champagnat. Finalmente, los resultados obtenidos tras la aplicación de la rúbrica y el proceso de observación directa permitieron concluir que a partir de las rúbricas es posible realizar una revisión rigurosa del nivel de comprensión en el que se encuentran los estudiantes para cada una de las temáticas trabajadas, lo cual permite utilizarlas tanto en los procesos de heteroevaluación como en los de autoevaluación, identificando fortalezas y oportunidades de mejoramiento en sus procesos de comprensión.

\section{Metodología}

El tipo de investigación realizado que se presenta en la (figura 1), tiene un enfoque en primer lugar, cualitativo, pues se observaron y describieron las características de la población entorno a los procesos de evaluación y autoevaluación que se efectuaban, sus perspectivas y las interpretaciones que se desarrollaron en torno a la elaboración y la realización de la rúbrica de evaluación. Por otra parte, es importante ligar este tipo de investigación a un segundo enfoque de carácter cuantitativo, pues se logró establecer un valor estadístico que permite acoplar los resultados obtenidos en la rúbrica de autoevaluación a la escala de valoración propuesta en el SIEE del Colegio Champagnat.

La población elegida para la realización del proyecto fueron los 101 estudiantes de grado $10 .^{\circ}$ del Colegio Champagnat en Bogotá. Con ellos se realizaron 
durante la práctica los procesos de observación, acompañamiento e intervención de clase.

Para el diseño de la rúbrica de evaluación (tabla 1) se tuvieron en cuenta las temáticas trabajadas en grado $10 .^{\circ}$ durante el cuarto periodo, como fue nomenclatura inorgánica, leyes de la conservación de la materia, tipos de reacciones y método de balanceo por tanteo; estas temáticas se encuentran ubicadas en la primera columna de la rúbrica de evaluación. Para la escala de valoración, se tomó la propuesta por el SIEE del Colegio Champagnat: en primer lugar, un nivel principiante valorado cuantitativamente entre $(1,0-6,9)$; posteriormente un nivel intermedio entre $(7,0-8,9)$ y avanzado entre ( 9,0 y 10$)$; finalmente, se encuentra una casilla de puntaje donde se asigna la valoración final total. Estas valoraciones se encuentran ubicadas en la primera fila de la rúbrica de evaluación. Para cada una de las temáticas se establecieron criterios respecto al nivel de comprensión que se consideraría debía tener el estudiante en cada uno de las valoraciones; la categoría principiante incluye los conceptos básicos que debería comprender, y la capacidad de comprensión que abarca todos los conceptos trabajados para la temática corresponde a la categoría avanzado (tabla 1). La evaluación se elaboró a partir de las mismas temáticas.

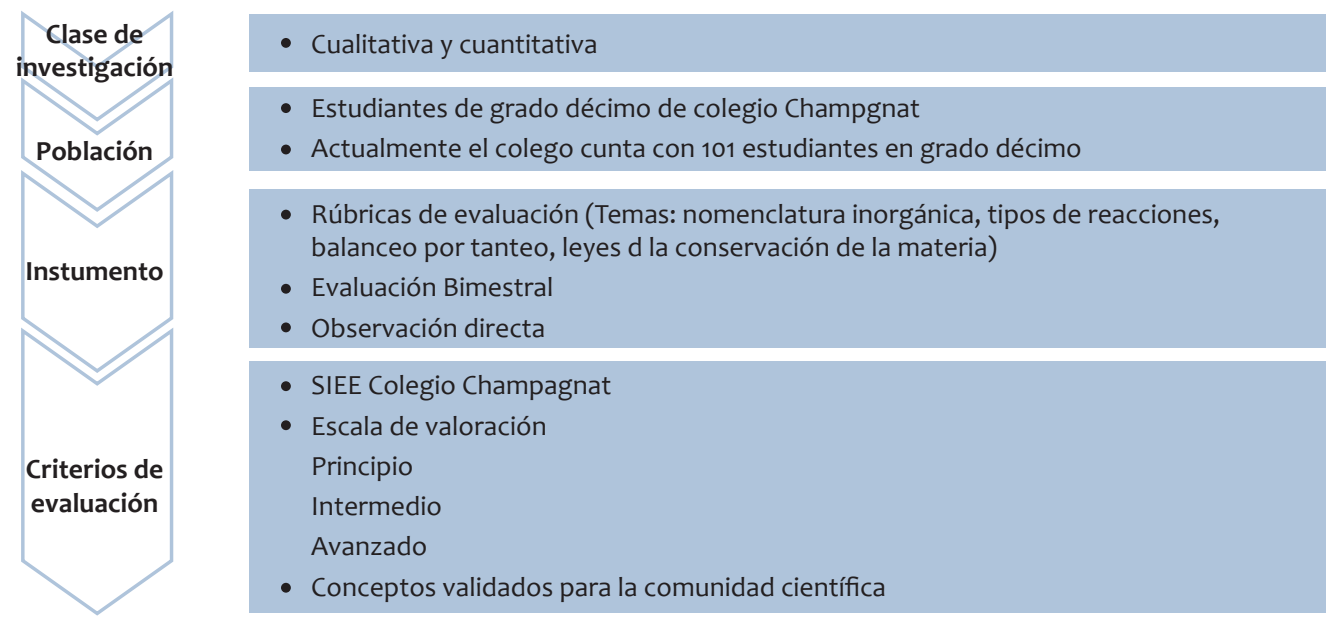

Figura 1. Diseño metodológico

Tabla 1. Rúbrica de evaluación y autoevaluación

\begin{tabular}{|c|c|c|c|c|}
\hline Aspecto a evaluar & Principiante (1.0-6.9) & Intermedio (7.0-8.9) & Avanzado (9.0-10) & Puntaje \\
\hline \multirow[t]{2}{*}{$\begin{array}{l}\text { Nomenclatura } \\
\text { inorgánica }\end{array}$} & $\begin{array}{l}\text { Identifica los estados de } \\
\text { oxidación de los elementos } \\
\text { y los utiliza correctamente } \\
\text { al momento de formar sus } \\
\text { respectivos iones, óxidos, } \\
\text { peróxidos y bases. }\end{array}$ & $\begin{array}{c}\text { Identifica los estados de } \\
\text { oxidación de los elementos } \\
\text { y los utiliza correctamente } \\
\text { al momento de formar sus } \\
\text { respectivos iones, óxidos, } \\
\text { peróxidos, bases, hidruros } \\
\text { y ácidos }\end{array}$ & $\begin{array}{c}\text { Identifica los estados de } \\
\text { oxidación de los elementos } \\
\text { y los utiliza correctamente } \\
\text { al momento de formar sus } \\
\text { respectivos iones, óxidos, } \\
\text { peróxidos, bases, hidruros, } \\
\text { ácidos y sales }\end{array}$ & \\
\hline & $\begin{array}{c}\text { Comprende la estructura } \\
\text { que caracteriza a los óxidos } \\
\text { ácidos y básicos, peróxidos } \\
\text { y bases }\end{array}$ & $\begin{array}{c}\text { Comprende la estructura } \\
\text { que caracteriza a los óxidos } \\
\text { ácidos y básicos, peróxidos, } \\
\text { bases, hidruros, ácidos oxá- } \\
\text { cidos e hidrácidos }\end{array}$ & $\begin{array}{l}\text { Comprende la estructura } \\
\text { que caracteriza a los óxidos } \\
\text { y básicos, peróxidos, bases, } \\
\text { hidruros, ácidos ozácidos e } \\
\text { hidrácidos, sales haloideas } \\
\text { y oxísales }\end{array}$ & \\
\hline
\end{tabular}




\begin{tabular}{|c|c|c|c|c|}
\hline Aspecto a evaluar & Principiante (1.0-6.9) & Intermedio (7.0-8.9) & Avanzado (9.0-10) & Puntaje \\
\hline \multirow[t]{4}{*}{$\begin{array}{l}\text { Nomenclatura } \\
\text { inorgánica }\end{array}$} & $\begin{array}{c}\text { Distingue la nomenclatura } \\
\text { stock que caracteriza a los } \\
\text { iones, óxidos, peróxidos y } \\
\text { bases }\end{array}$ & $\begin{array}{l}\text { Distingue la nomenclatura } \\
\text { stock que caracteriza a } \\
\text { los iones, óxidos ácidos y } \\
\text { básicos, peróxidos, bases, } \\
\text { hidruros, ácidos xácidos e } \\
\text { hidrácidos. }\end{array}$ & $\begin{array}{c}\text { Distingue la nomenclatura } \\
\text { stock que caracteriza a } \\
\text { los iones, óxidos ácidos y } \\
\text { básicos, peróxidos, bases, } \\
\text { hidruros, ácidos oxácidos e } \\
\text { dirácidos, salees haloideas } \\
\text { y oxísales }\end{array}$ & \\
\hline & $\begin{array}{c}\text { Distingue la nomenclatura } \\
\text { sistemática que caracteriza } \\
\text { a los iones, óxidos, peróxi- } \\
\text { dos y bases }\end{array}$ & $\begin{array}{l}\text { Distingue la nomenclatura } \\
\text { sistemática que caracteriza } \\
\text { los iones, óxidos ácidos y } \\
\text { básicos, peróxidos, bases, } \\
\text { hidruros, ácidos oxácidos e } \\
\text { hidrácidos }\end{array}$ & $\begin{array}{c}\text { Distingue la nomenclatura } \\
\text { sistemática que caracteriza } \\
\text { a los iones, óxidos ácidos } \\
\text { y básics, peróxidos, bases, } \\
\text { hidruros, ácidos, oxácidos } \\
\text { hidrácdos, sales haloideas y } \\
\text { oxísales }\end{array}$ & \\
\hline & $\begin{array}{c}\text { Distingu la nomenclatura } \\
\text { tradicional que caracteriza } \\
\text { a los iones, óxids, peróxidos } \\
\text { y bases }\end{array}$ & $\begin{array}{l}\text { Distingue la nomenclatura } \\
\text { tradicional que caracteriza } \\
\text { a los iones, oxidos ácidos y } \\
\text { básicos, peróxidos, bases, } \\
\text { hidruros, ácidos oxácidos e } \\
\text { hidrácidos }\end{array}$ & $\begin{array}{l}\text { Distingue la nomenclatura } \\
\text { tradicional que caracteriza } \\
\text { a los iones, óxidos ácidos y } \\
\text { básicos, peróxidos, bases, } \\
\text { hidruros, ácidos oxácidos e } \\
\text { hidrácidos, sales haloideas } \\
\text { y oxísales }\end{array}$ & \\
\hline & $\begin{array}{l}\text { Escribe correctamente la } \\
\text { formula mlecuar partiendo } \\
\text { de la nomenclatura que } \\
\text { caracteriza a los iones, óxi- } \\
\text { dos, peróxidos y bases }\end{array}$ & $\begin{array}{c}\text { Escribe correctamente } \\
\text { la formula molecular } \\
\text { partiendo de la nomen- } \\
\text { clatura que caracteriza a } \\
\text { los iones, óxidos ácidos y } \\
\text { básicos, peróxidos, bases, } \\
\text { hidruros, ácidos oxácidos e } \\
\text { hidrácidos. }\end{array}$ & $\begin{array}{l}\text { Escribe correctamente la } \\
\text { formula molecular par- } \\
\text { tiendo de la nomenclatura } \\
\text { que caracteriza a los iones, } \\
\text { óxidos ácidos y básicos, } \\
\text { persóxidos, bases, hidruros, } \\
\text { ácidos oxácidos e hidrá- } \\
\text { cidos, sales haloideas y } \\
\text { oxísales }\end{array}$ & \\
\hline \multirow[t]{3}{*}{$\begin{array}{l}\text { Reacciones } \\
\text { químicas }\end{array}$} & $\begin{array}{l}\text { Identifica los tipos de } \\
\text { reacciones químicas inor- } \\
\text { gánicas, síntesis, descom- } \\
\text { posición desplazamiento y } \\
\text { doble desplazamiento }\end{array}$ & $\begin{array}{l}\text { Diferencia los tipos de } \\
\text { reacciones químicas inor- } \\
\text { gánicas, síntesis, descom- } \\
\text { posición desplazamiento y } \\
\text { doble desplazamiento en } \\
\text { las diferentes ecuaciones } \\
\text { planteadas }\end{array}$ & $\begin{array}{c}\text { Clasifica los tipos de } \\
\text { reacciones químicas inor- } \\
\text { gánicas, síntesis, descm- } \\
\text { posición, desplazamiento } \\
\text { y doble desplazamiento } \\
\text { que se presentan en la } \\
\text { naturaleza }\end{array}$ & \\
\hline & $\begin{array}{c}\text { Reconoce las ecuaciones } \\
\text { químicas como la repre- } \\
\text { sentación de una reacción } \\
\text { química }\end{array}$ & $\begin{array}{c}\text { Formula ecuaciones quími- } \\
\text { cas a partir de las reaccio- } \\
\text { nes químicas ppresentadas } \\
\text { durante las prácticas de } \\
\text { laboratorio }\end{array}$ & $\begin{array}{l}\text { Explica la formación de nue- } \\
\text { vas sustancias en términos } \\
\text { de reactantes y productos }\end{array}$ & \\
\hline & $\begin{array}{l}\text { Reconoce en un reacción } \\
\text { química, la Ley de la Conser- } \\
\text { vación de la Materia }\end{array}$ & $\begin{array}{l}\text { Relacionar las ecuaciones } \\
\text { químicas con la ley de la } \\
\text { conservación de la materia } \\
\text { en ejercicios sencillo balan- } \\
\text { ceándolos por el método } \\
\text { de tanteo }\end{array}$ & $\begin{array}{l}\text { Comprueba en las ecuacio- } \\
\text { nes químicas los coeficien- } \\
\text { tes correctos que determi- } \\
\text { nan la principal ley de esta } \\
\text { ciencia experimental, la } \\
\text { conservación de masa, utili- } \\
\text { zando el método de tanteo }\end{array}$ & \\
\hline \multicolumn{5}{|c|}{ TOTAL } \\
\hline
\end{tabular}


Para el análisis de los resultados, se tuvo en cuenta la evaluación diagnóstica y el proceso de autoevaluación a partir de los criterios establecidos en la rúbrica. Adicionalmente, se realizó un proceso de observación directo al momento del desarrollo de las rúbricas de autoevaluación para conocer las perspectivas de los estudiantes respecto a su proceso de comprensión desarrollado durante el periodo.

Los resultados obtenidos en la evaluación diagnóstica y la rúbrica de autoevaluación desarrollada por los estudiantes se presentan a continuación (tabla 2 y figura 2 ).

Tabla 2. Resultados evaluación diagnóstica

\begin{tabular}{|c|c|c|c|c|}
\hline & \multicolumn{3}{|c|}{ Evaluación bimestral } & \\
\hline & $\begin{array}{c}\text { Princi- } \\
\text { piante }\end{array}$ & $\begin{array}{c}\text { Interme- } \\
\text { dio }\end{array}$ & Avanzado & total \\
\hline $\begin{array}{c}\text { Número } \\
\text { de estu- } \\
\text { diantes }\end{array}$ & $50,49 \%$ & $36,63 \%$ & $11,88 \%$ & $100 \%$ \\
\hline
\end{tabular}

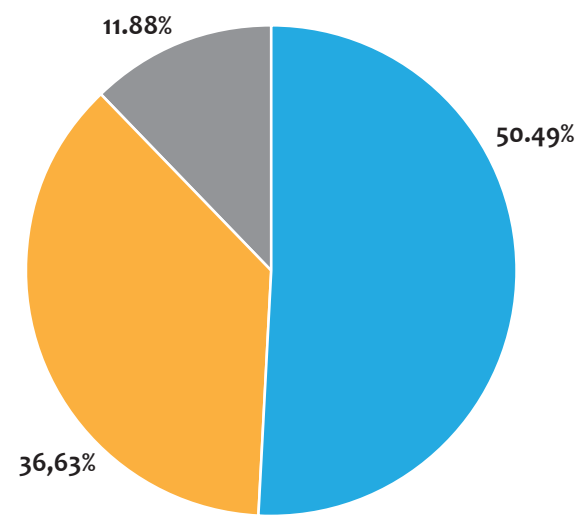

Figura 2. Evaluación diagnóstica

\section{Resultados evaluación diagnóstica}

El 50,49\% de los estudiantes se encuentra en un nivel principiante según la prueba diagnóstica aplicada y los criterios planteados en la rúbrica de evaluación. Los estudiantes reconocen la nomenclatura stock tradicional y sistemática que caracteriza a los óxidos, peróxidos y bases; identifican los tipos de reacciones químicas inorgánicas de síntesis, descomposición y desplazamiento, reconociendo las ecuaciones químicas, como la representación de una reacción química, en la que existe una ley de la conservación de la materia.

Se puede observar que un 36,63\% de los estudiantes se encuentra en un nivel intermedio según la prueba diagnóstica aplicada y los criterios planteados en la rúbrica de evaluación. Estos estudiantes identifican y clasifican algunos de los tipos de nomenclatura que caracterizan a los iones, óxidos ácidos y básicos, peróxidos, bases, hidruros, ácidos oxácidos e hidrácidos. Por otra parte, diferencian los tipos de reacciones químicas inorgánicas, síntesis, descomposición desplazamiento y doble desplazamiento en los diferentes ejercicios planteados, formulan ecuaciones a partir de las reacciones químicas presentadas durante las prácticas de laboratorio y relacionan las ecuaciones químicas con la ley de la conservación de la materia.

Un $11,88 \%$ de los estudiantes se encuentra en un nivel avanzado. Ellos identifican, comprenden explican y clasifican los tipos de nomenclatura que caracterizan a los iones, los óxidos ácidos y los básicos, los peróxidos, las bases, los hidruros, los ácidos oxácidos y los hidrácidos, las sales haloideas y las oxisales. También clasifican los tipos de reacciones químicas inorgánicas, síntesis, descomposición desplazamiento y doble desplazamiento que se presentan en la naturaleza. De igual manera, explican la formación de nuevas sustancias en términos de reactantes y productos, balancean las ecuaciones químicas con los coeficientes correctos que determinan la principal ley de esta ciencia experimental, la conservación de masa, y utilizan el método de tanteo.

\section{Resultados de la autoevaluación}

Tabla 3. Resultados rúbrica autoevaluación

\begin{tabular}{|c|c|c|c|c|}
\hline & \multicolumn{3}{|c|}{ Autoevaluación } & \\
\hline & $\begin{array}{c}\text { Princi- } \\
\text { piante }\end{array}$ & $\begin{array}{c}\text { Interme- } \\
\text { dio }\end{array}$ & Avanzado & total \\
\hline $\begin{array}{c}\text { Número } \\
\text { de estu- } \\
\text { diantes }\end{array}$ & $14,85 \%$ & $49,50 \%$ & $35,64 \%$ & $100 \%$ \\
\hline
\end{tabular}




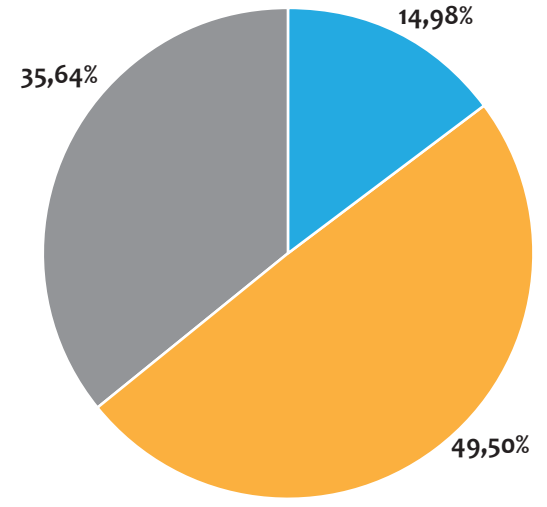

Figura 3. Autoevaluación

La autoevaluación muestra que un $14,85 \%$ de la población evaluada se encuentra en un nivel principiante: como inicialmente en este nivel se encontraba el 50,49\% de los estudiantes, se infiere que el $36,63 \%$ de ellos se ubicaron en niveles superiores a este. El 49,50\% de la población se ubicó en un nivel intermedio, lo que significa que aumentó en un $12,87 \%$ respecto a la evaluación diagnóstica. Finalmente, se evidencia que el porcentaje para el nivel avanzado se triplicó, pues de un $11,88 \%$ en la evaluación diagnóstica se pasó a tener un $35,64 \%$ de estudiantes para este nivel. Se deduce un avance en la comprensión de los conceptos de reacción química, aplicación de la ley de conservación de la masa al mejorar los procesos de balance por inspección o tanteo, y el reconocimiento de las funciones inorgánicas y su nomenclatura.

Al comparar el resultado de la evaluación y la autoevaluación se evidencia que el $63,37 \%$ de los estudiantes muestra una relación coherente al identificar el nivel de comprensión alcanzado durante la clase de química para los temas de reacciones químicas y nomenclatura inorgánica con los resultados de la evaluación bimestral. Este porcentaje de estudiantes logró identificar falencias y fortalezas en sus procesos de comprensión mediante la implementación de la rúbrica, lo que permitirá proponer estrategias que mejoren sus procesos de comprensión de los conceptos químicos
Para el $35,64 \%$ restante no se evidencia una correlación entre los resultados obtenidos en el proceso de evaluación y autoevaluación, porcentaje de estudiantes que sigue considerando el proceso de autoevaluación una estrategia para mejorar su promedio.

La rúbrica de evaluación, al establecer criterios claros frente a las temáticas a evaluar, permitió que tanto el estudiante como el docente tuvieran claras las temáticas que se abordaron durante el periodo y los parámetros según los cuales se estaba evaluando su proceso de autoevaluación, como la prueba diagnóstica.

\section{Conclusiones}

Mediante la rúbrica se realizó un proceso riguroso del nivel de comprensión en el que se encontraban los estudiantes para cada una de las temáticas trabajadas, en los procesos tanto de heteroevaluación como de autoevaluación. Se pudo ratificar que, con ayuda de las rúbricas de evaluación y los criterios allí planteados los estudiantes, pudieron identificar el nivel de comprensión que habían alcanzado en las temáticas trabajadas durante el semestre.

La implementación de la rúbrica durante el proceso de autoevaluación realizado por los estudiantes, de grado $10 .^{\circ}$ en las clases de química, le permitió al $63,37 \%$ de la población identificar oportunidades de mejoramiento y fortalezas en sus procesos de comprensión de los conceptos químicos, lo que permitirá proponer estrategias que mejoren dichos procesos. También posibilitó el progreso de los estudiantes de grado $10 .^{\circ}$ en su proceso de autoevaluación como medio para identificar su avance en la comprensión de conceptos químicos.

\section{Referencias}

Stone Wiske, M. (1999). La enseñanza para la comprensión. Buenos Aires: Paidós. 
